similar treatments for the control of somatic diseases. The fact of imagining a scene where oneself is the one suffering from a disease, shows preferences in the use of psychotropic drugs for the management of schizophrenia where the profile of side-effects and efficacy has a more equitable balance: starting from comparable effectiveness, we prefer treatments associated with a perception of fewer sideeffects

19

\section{Real World Patient-Reported Outcomes Following Pharmacogenomic Testing}

Nichole Rigby, MS ${ }^{\prime}$; Jennifer $\mathrm{Ma}^{\prime}$; Joseh Boland'; Danile Van Dorm, PharmD ${ }^{\prime}$; Daniel Dowd, PharmD'; and David Krause, $M D^{\prime}$

${ }^{1}$ Genomind, King of Prussia, PA

ABSTRACT: Background: The use of pharmacogenomics (PGx) testing has the potential to accelerate response to psychopharmacologic therapy $(\mathrm{Rx})$ and improve outcomes; accordingly PGx use to select appropriate $\mathrm{Rx}$ is increasing. One such commercially available test is the Genecept Assay (the Assay [Genomind]) which measures variants of 18 genes (12 pharmacodynamic and 6 CYP450) for which response, tolerability or exposure to various $R x$ has been reported. Recent interest in genetics has led patients (pts) to be stewards of their own genetic data. In 2017 we launched a Patient Gateway to allow pts to retrieve their genetic results, have access to mental health information, and record outcomes following use of the Assay.

OBJECTIVE: To assess the effectiveness of $\mathrm{Rx}$ recommendations following use of the Assay, as reported on a purpose-built patient portal.

METHOD: Pts receiving the Assay were invited to visit an online, interactive portal. Pts providing informed consent (IC) were asked to record their baseline overall health using a 4- point modified patient global index (mPGI) of severity. Pts also recorded their conditions, medications, and supplements, and various symptoms. Pts were invited to visit the portal ad libitum and re-rate their overall health using the m-PGI. These data were then combined with the pts' genetic results using custom scripts in Python (v 3.6.4) and R (v 3.5.1). All identifying data were removed. Pts included in this analysis responded (at least) twice to the health questionnaire. New medications were subsequently scored as concordant, discordant, or indeterminate with the Assay's recommendations, using predetermined criteria. We report the initial results for this subgroup herein.
RESULTS: Since launch 9,401 unique patient profiles were created on the Gateway; 5,207 (55\%) of these provided IC. Of these, 410 provided at least 2 m-PGI scores. Seventy-three (73) of these pts reported scores at least 4 weeks apart and started 222 medications in the interim. $69.4 \%$ of pts identified as female; $70.8 \%$ had a diagnosis of generalized anxiety disorder, while $50.0 \%$ and $31.9 \%$ had diagnoses of major depressive disorder and post-traumatic stress disorder, respectively. $60.2 \%$ of pts reported improvement on the m-PGI of $\geq 1$ unit; $20 \%$ had a $\geq 2$-unit improvement. Pts reporting improvement were more likely $(77 \%$ vs $66 \%)$; to have been placed on medication that were concordant with the assay than those who were not improved, although this difference did not reach statistical significance.

CONCLUSION: In this naturalistic, virtual trial of a PGx assay to guide pharmacotherapy in individuals with mental health illness, most users reported improvement in overall health. More pts whose medication was reported as concordant with the Assay reported improvement than those with discordant medications. Data collection is ongoing and updated data will be provided. Funding Acknowledgements: Genomind

\section{0 \\ The Need for Speed: Adjunctive Triple Chronotherapy in the Accelerated Treatment of Acute Depression and Suicidality in the Adolescent Population}

Diane Hurd, PMHNP'; Eric Arzubi, MD' ${ }^{2}$; Mariela Rojas Herrera, $\mathrm{MD}^{3}$; Nicholas Coombs, $\mathrm{MS}^{4}$; and Jeannine Brant, PhD, APRN, AOCN, FAAN

${ }^{1}$ Psychiatric Nurse Practitioner, Behavioral Health Center, Billings Clinic, Billings, MT

${ }^{2}$ Child Adolescent Psychiatrist, Chair of Psychiatry, Billings Clinic, Billings, MT

${ }^{3}$ Child Adolescent Psychiatrist, Director of the Psychiatric Youth Treatment Unit, Billings Clinic, Billings, MT

${ }^{4}$ Data Analyst, Collaborative Science \& Innovation, Billings Clinic, Billings, MT

${ }^{5}$ Nurse Scientist, Collaborative Science and Innovation, Billings Clinic, Billings MT

ABSTRACT: Objective: This pilot study aims to explore the feasibility and proof of concept of triple chronotherapy (TCT) as a non-pharmacological, adjunctive intervention in the treatment of acute depression and suicidality in the adolescent population.

METHOD: Thirty-one adolescents with moderate to severe depression were included in the study. Each participant 